\title{
Retro-Odontoid Calcium Pyrophosphate Dehydrate Deposition: Surgical Management and Review of the Literature
}

\author{
Eric Klineberg ${ }^{1}$ Tuan Bui ${ }^{2}$ Richard Schlenk ${ }^{3}$ Isador Lieberman ${ }^{4}$
}

${ }^{1}$ Department of Orthopaedic Surgery, University of California Davis School of Medicine, Sacramento, California, United States

2 Department of Orthopaedics, Drexel University, Philadelphia, Pennsylvania, United States

${ }^{3}$ Cleveland Clinic Foundation, Cleveland, Ohio, United States

${ }^{4}$ Scoliosis and Spine Tumor Center, Texas Back Institute, Plano, Texas, United States

Evid Based Spine Care J 2014;5:63-69.
Address for correspondence Eric Klineberg, MD, Department of Orthopaedics, Adult and Pediatric Spinal Surgery, 4860 Y Street, Suite 3800, Sacramento, CA 95817, United States

(e-mail: eric.klineberg@ucdmc.ucdavis.edu).

\author{
Abstract \\ Keywords \\ - crowned dens \\ syndrome \\ - calcium \\ pyrophosphate \\ dihydrate \\ - retro-odontoid mass \\ - cervical myelopathy \\ - crystal deposition \\ disease \\ - cervical \\ decompression \\ - cervical mass \\ aspiration
}

Study Design Case report and review of the literature.

Objective A retro-odontoid mass is a rare cause of cervical compression and myelopathy. The differential diagnosis includes the following: metastatic disease, primary tumor, collagen disorder, or inflammatory disease. Calcium pyrophosphate dihydrate (CPPD) deposition has been referred to as "crowned dens syndrome" when there are periodontoideal calcifications. There are only a few reported cases where CPPD presents as a cystic retro-odontoid mass in the atlanto-dens interval. In previous descriptions of surgical intervention, transoral resection of the mass is associated with significant morbidity and usually requires stabilization. The objective of this article is to report a case of an unusual presentation of CPPD disease of C1/C2, where we used a novel, minimally invasive surgical technique for decompression without fusion.

Patients and Methods An 83-year-old female patient presented with progressive cervical myelopathy over a 3-month period. Computed tomography and magnetic resonance imaging demonstrated a cystic odontoid mass with a separate retro-odontoid compressive mass. A novel, minimally invasive transoral aspiration was performed. Histologic confirmation of CPPD was obtained.

Results Postop imaging showed satisfactory decompression, which was maintained at the 6-month follow-up. This correlated with clinical improvement postop and 6-month follow-up.

Conclusion CPPD in the atlanto-dens interval may present as a cystic retro-odontoideal mass and should be included in the differential. We used a transoral minimally invasive approach to aspirate the cyst. This novel technique avoided the need for a stabilization procedure or morbid transoral resection and provided excellent results immediately and at 6 months. (c) 2014 Georg Thieme Verlag KG Stuttgart · New York
DOI http://dx.doi.org/ 10.1055/s-0034-1370897. ISSN 1663-7976. 

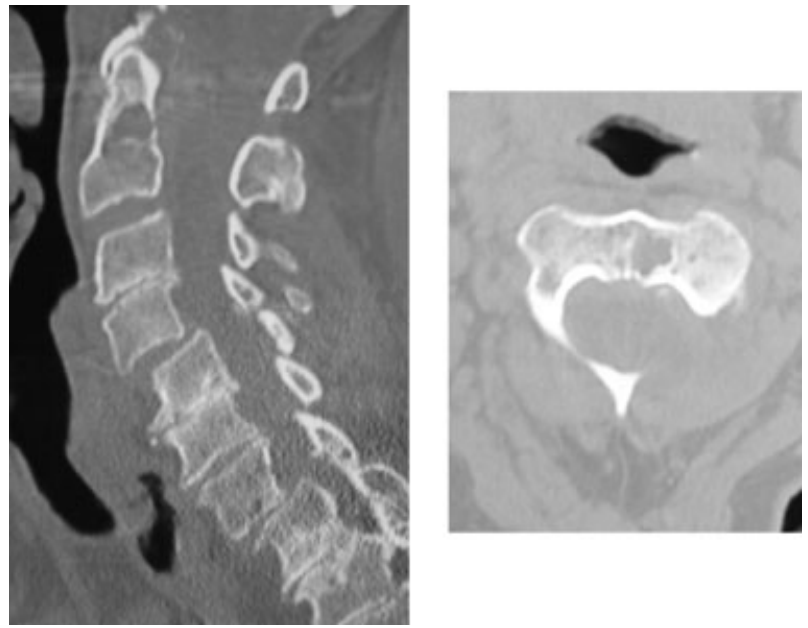

Fig. 1 Sagittal and coronal computed tomography scan demonstrating cystic mass at the base of the dens, with multilevel cervical spondylosis.

\section{Introduction}

Calcium pyrophosphate dihydrate (CPPD) deposition in the cervical spine is an unusual cause of cervical spinal cord compression and myelopathy. Retro-odontoideal crystal deposits may lead to acute pseudogout and can be visualized as calcifications that surround the apex of the dens, and is therefore known as "crowned dens syndrome" (CDS). ${ }^{1,2}$ This entity may manifest itself in a variety of ways, but it usually presents as acute neck pain and stiffness., ${ }^{3,4}$ With disease progression, it may present as a mass-occupying lesion, causing progressive cervical spinal cord compression and myelopathy. In general, noninfectious, nontumorous retro-odontoid cystic masses are exceedingly rare. ${ }^{5}$ They have mainly been described in the literature as synovial cysts, ganglion cysts, transverse ligament degeneration, or herniated discs. ${ }^{5,6}$
We present a rare case of retro-odontoid CPPD resulting in cervical myelopathy that required surgical intervention. Our patient is an 83-year-old woman with a cystic lesion within the body of the dens, as well as a retro-odontoid mass, causing cervical spinal cord compression. She had no history of pseudogout, or other predisposing factors. We successfully decompressed her lesion using a minimally invasive transoral decompression, under fluoroscopic visualization. The literature on cervical CPPD was reviewed, and our surgical approach discussed.

\section{Case Report}

An 83-year-old female patient presented to our emergency department (ED) with progressive difficulty with balance and upper extremity clumsiness. While the balance difficulty had been progressive for some time, it had worsened drastically in the few weeks before presentation in the ED. Medical history is negative for diabetes or rheumatoid disease. There was no history for joint swelling or inflammation in any of her major joints.

Physical examination revealed hyperreflexia in her upper and lower extremities with bilateral positive Hoffman and pathological clonus. She had diffuse weakness in her upper and lower extremities suggestive of neural compression. She also had diffuse paresthesias that was nondermatomal.

Computed tomography (CT) and magnetic resonance imaging (MRI) scans were completed to determine the etiology of her myelopathy. The CT scan revealed multilevel spondylosis without evidence of acute fracture or instability, and with a cystic lesion located within the body of the dens (-Fig. 1). This lesion was noted to be hyperintense on T2weighted MRI and to be associated with a large extradural mass. The mass caused significant cord compression, and was also associated with a bilobulated cystic mass that resulted in additional anterior compression, best appreciated on the axial MRI (-Fig. 2).
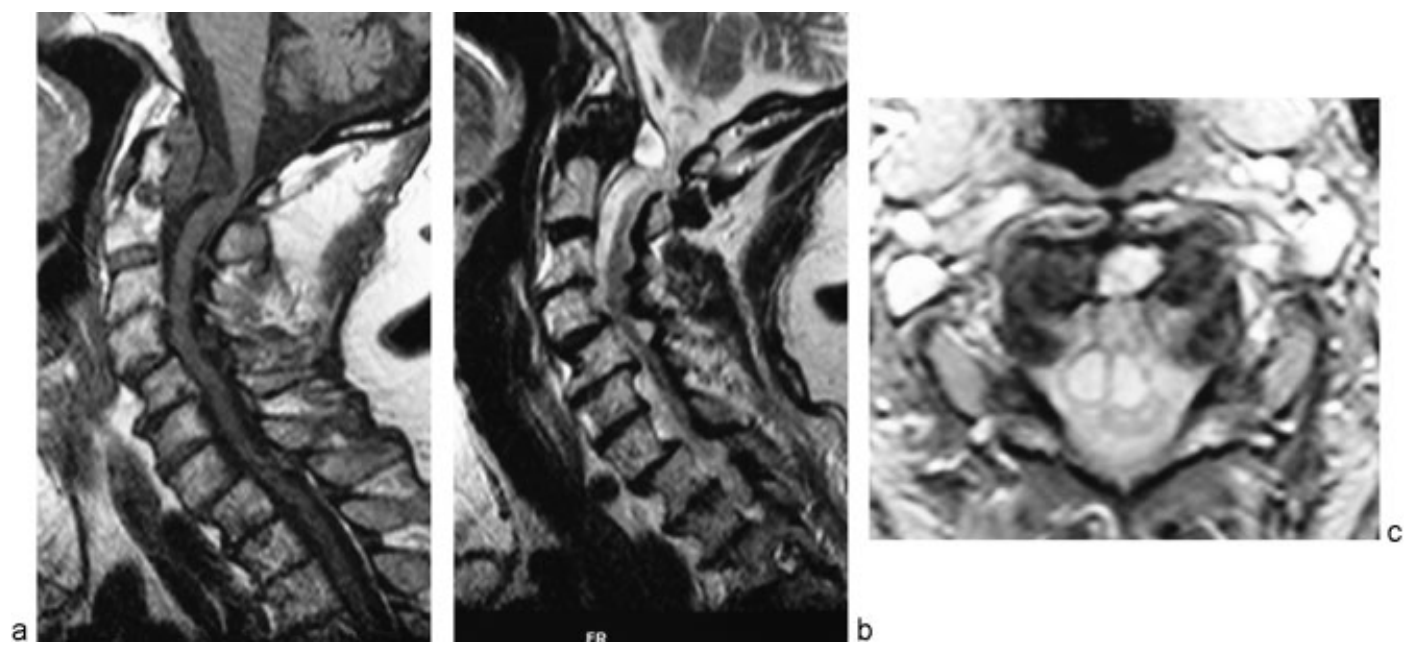

Fig. 2 Magnetic resonance imaging ( $\mathrm{T} 1$ (a) and $\mathrm{T} 2$ (b, c) sequences) demonstrating a retro-odontoid compressive mass with additional bilobulated cystic compression, with myelomalacia noted in the cord. 


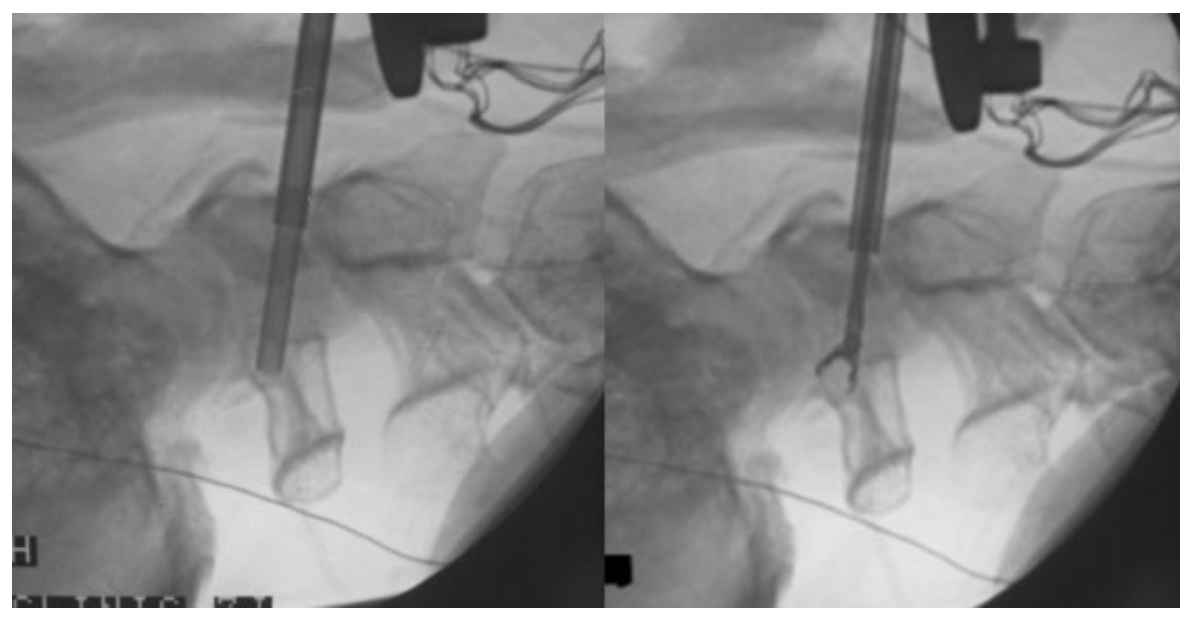

Fig. 3 Intraoperative fluoroscopic images of the sleeved trocar placed through the retro-odontoid mass and into the cyst, and removal of the cystic material.

As our patient had progressive neurologic decline, we felt that surgical decompression was indicated. As this was an elderly 83-year-old patient, we were concerned about the morbidity of either a posterior fusion followed by an anterior transoral approach or a posterior fusion alone. After a lengthy discussion with the patient and her family, we elected to proceed with an anterior minimally invasive transoral aspiration of the cystic lesion to determine the etiology and to decompress the spinal cord within the canal.

\section{Technique}

The patient was positioned and intubated supine and we used the Crockard Transoral Instrumentation System to maintain the exposure (Codman \& Shurtleff, Inc., Raynham, MA, United States). The uvula was reflected out of the way using a suture and red rubber catheter through the nasopharyngeal cavity. The retropharyngeal soft tissue was visualized, and using computer-

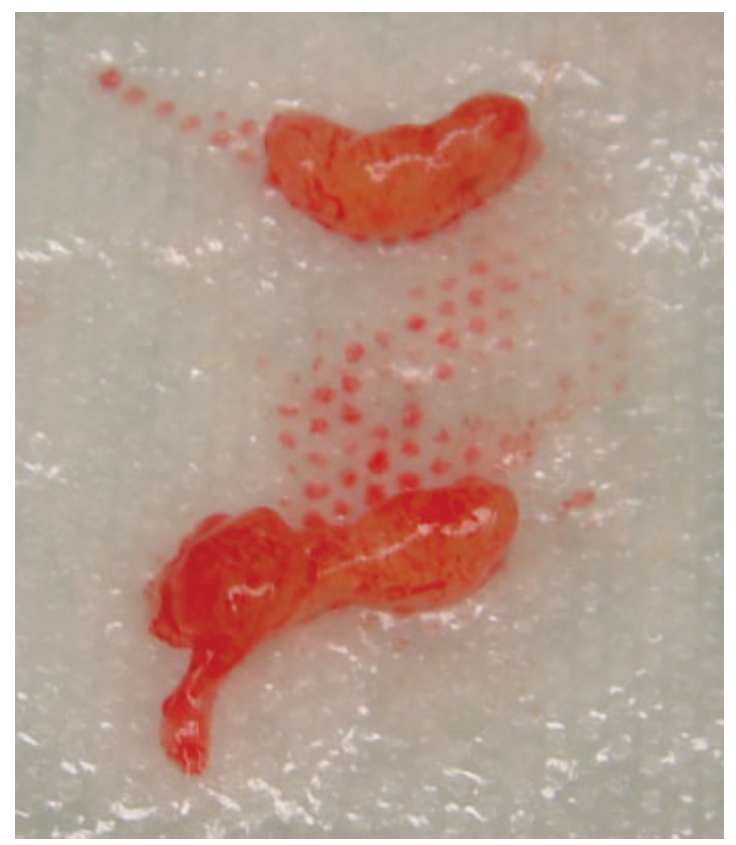

Fig. 4 Gross specimen: gelatinous material removed from cyst. assisted navigation, the correct trajectory and entry point was selected. A small 5-mm incision was made into the retropharyngeal tissue anterior to the body of C2. Then using fluoroscopy, a trochar was advanced into and through the body of $\mathrm{C} 2$ into the retro-odontoid pannus ( - Fig. 3 ). The two cysts were then sequentially aspirated. Two gelatinous aspirates were removed and sent for pathology ( - Fig. 4). Histology revealed inflammatory cells with positively birefringent rhomboid crystals, consistent with the diagnosis of CPPD. The trochar was removed and the retropharynx closed primarily. Immediate postoperative MRI revealed successful cyst aspiration (- Fig. 5).

The patient's postoperative course was complicated by respiratory distress that required reintubation. This resolved with supportive care and the patient was discharged home in stable condition, with resolution of her myelopathy including improved strength and sensation. At 6-month follow-up, the patient was walking with a cane only, and showed continued functional improvement. A repeat MRI at that time revealed complete resolution of the cysts and improved spinal canal diameter (-Fig. 6).
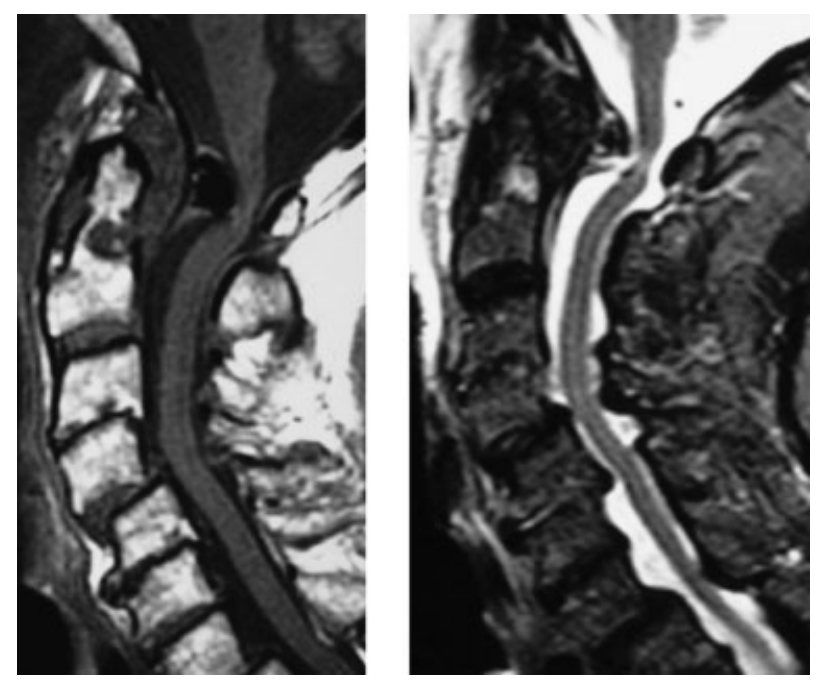

Fig. 5 Immediate postoperative magnetic resonance imaging (MRI), demonstrating successful cyst aspiration. Note the absence of signal on T2-weighted MRI (right). 

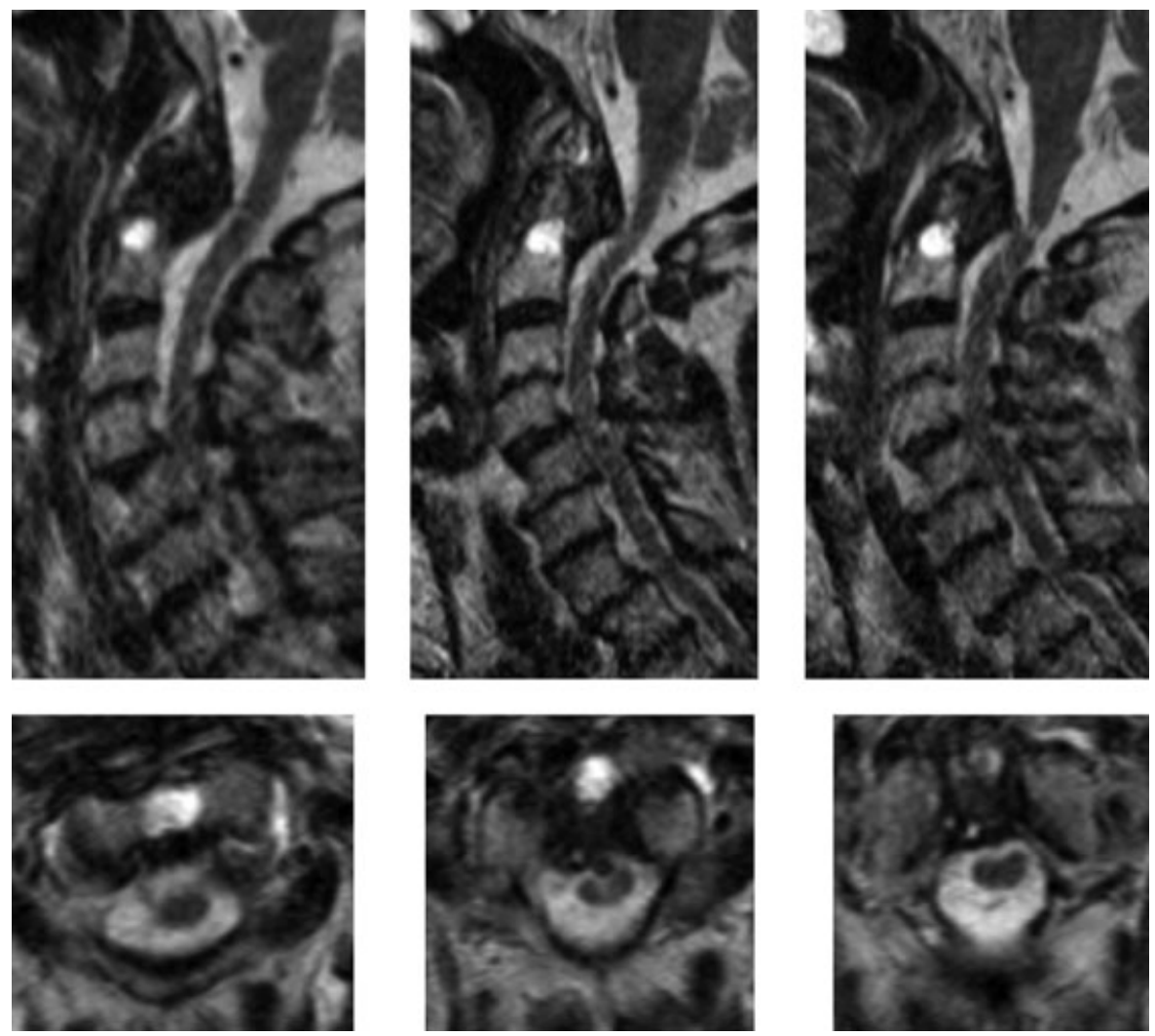

Fig. 6 Six-month postoperative magnetic resonance imaging, axial and sagittal T2 sequences. Note the absence of cyst on images, and significant improvement in cord compression.

\section{Discussion}

Cervical myelopathy that occurs as a result of compression at the craniocervical junction is uncommon and may be difficult to diagnose. The etiology of the compression may include rheumatic disease, craniocervical tumors, synovial cyst and CPPD deposition. There are increasing numbers of case reports regarding CPPD deposition at the $\mathrm{C} 1 / 2$ articulation. Although more likely at advanced ages, in 2009, Unlu et al described a case of idiopathic CDS in a young male, causing severe neck pain. ${ }^{7,8}$ Clearly, the diagnosis of CPPD should be considered in all patient populations, and may occur in the absence of significant risk factors.

Most cases of CDS arise in patients with known CPPD disease. In 2001, Ryan et al noted that there was asymptomatic calcification of the periodontoid region in half of patients with a known diagnosis of chondrocalcinosis, "suggesting a high incidence of involvement of these structures in CPPD disease." 9 Interestingly, they noted that this deposition did not cause clinical disease in any of their patients. A 2004 case-control study by Finckh et al described that cervical calcifications were found in $69 \%$ of CPPD patients ( 24 of 35 ), compared with only $11 \%$ in controls. In addition, these patients were five times more likely to report neck pain. ${ }^{10}$ In 2008, Salaffi et al had similar results with $51 \%$ (25 of 49 ) having periodontoid calcifications in known CPPD. They continued further to describe subchondral cysts or erosion in $40 \%$ (10 of 25 ) of those patients. ${ }^{3}$ When clinically significant, the crystal deposition can present either acutely or insidiously, and mimics an inflammatory process. ${ }^{6}$ The correct diagnosis may be difficult, as CPPD deposition can occur at any location in the spine. Resnick et al conducted a postmortem study of more than 1,000 spines and found CPPD deposition in the intervertebral discs, interspinous and supraspinous ligaments, ligamentum flavum, posterior longitudinal ligament, sacroiliac joint, transverse atlas ligament, and posterior median atlantoaxial joint. ${ }^{10,11}$ There are numerous case reports showing CPPD in various locations in the spine, supporting this.

Atlantoaxial CPPD deposition is best visualized on CT and plain film. ${ }^{1,12}$ It was initially described by Bouvet et al as the "crowned dens" in $1985 .{ }^{13}$ CT is generally considered the most sensitive modality of visualizing periodontoid calcifications, and the existing literature suggests that diagnosis of CPPD deposition is largely radiographic. ${ }^{14,15}$ The largest review of this syndrome was reported in The Journal of Bone and Joint Surgery in 2007 by Goto et al. ${ }^{16}$ They reviewed 40 patients retrospectively that had characteristic calcium deposits visualized on CT scan; however, none of these patients had histologic confirmation, and none displayed symptomatic disease.

In the English literature, a limited number of cases of CDS have had their diagnosis confirmed. In our case, the CPPD diagnosis was verified via histology, which was especially important due to the absence of significant calcium deposition on CT scan. A cystic odontoid mass, while also previously documented in CPPD, usually lends itself to an alternate diagnosis, including synovial or ganglionic cysts, and transverse ligament degeneration. ${ }^{3}$ We attained confirmation with the classic weakly positively birefringent rhomboid crystals 
on histological analysis. Our MRI findings were similar to others, with the compressing mass roughly isointense on T1weighted, and very hyperintense on T2-weighted. ${ }^{17-20}$ As with any abnormal presentation, we recommend thorough radiographic studies to rule out other diagnoses.

Treatment for CPPD is symptomatic, and certainly nonsurgical modalities, including medications, should be attempted before surgical intervention when possible, with management tailored to both disease and patient. Of the existing literature on cervical CPPD disease, only a small fraction of cases required surgical intervention due to either pain, myelopathy, or type-2 odontoid fracture. ${ }^{19,21}$ Retro-odontoid masses are difficult to reach safely with traditional surgical techniques. Posterior approaches are familiar but require manipulation of the vulnerable spinal cord. Whiteside approach allows access to the dens, but requires significant bony resection, and does not easily allow for instrumentation. A transoral approach allows for direct access from the anterior clivus down to $\mathrm{C} 3$ or $\mathrm{C} 4$. It also reduces manipulation of the nearby neurovascular structures, compared with posterior and lateral approaches. However, the approach introduces oral flora into the surgical field, increasing the risk of infection. Other complications include upper airway obstruction secondary to edema, velopalatine insufficiency, dental injury, tongue necrosis/edema, odynophagia, dysphagia, meningitis, pharyngeal cellulitis, and TMJ syndrome. ${ }^{22-24}$ All of these approaches have significant morbidity and may require surgical stabilization before or after anterior decompression. At the very least, they require a period of modified nutritional support and attention to aspiration and possible swallowing difficulties, as well as observation for potential infection. In 1996, Zünkeler et al described seven cases of histology-confirmed CPPD masses that were posterior to the odontoid process. These patients underwent a transoral-transpharyngeal resection of the anterior arch of $\mathrm{C} 1$, odontoid process, and the compressing mass. ${ }^{25}$ However, six of the seven patients required posterior fixation at a later time to treat instability. ${ }^{22}$

To minimize complications, different techniques have been used to reduce pharyngeal dissection, such as endoscopic-assisted and minimally invasive approaches. Our technique utilized a cannula to assist with decompression of the retroodontoid space. This minimizes the risks associated with a transoral approach, with the advantage of direct decompression of the mass. It also allows for resection of the mass with minimal bone resection, which may prevent late instability. The limitations are that this approach is technically demanding, is reliant on ideal intraoperative imaging, and requires a mass that can be removed via the cannula. Despite the previously stated limitations, this approach has proven to be a viable alternative for direct surgical aspiration through the transoral approach, and avoids the need for surgical stabilization.

\section{Conclusion}

While CPPD is a rare cause of cervical spinal cord compression, deposition may occur at a variety of locations in the craniocervical junction, and should be included in the differential diagnosis of cervical inflammatory diseases. ${ }^{26-31}$ Advanced imaging is required to correctly identify the etiology of the compression, and may offer some clues to the diagnosis, particularly if the dens is surrounded by calcifications. We present a case of CPPD in the retro-odontoid space that caused significant spinal cord compression. We utilized novel minimally invasive transoral aspiration to establish the diagnosis, and affect appropriate decompression with minimal morbidity.

\section{References}

1 Scutellari PN, Galeotti R, Leprotti S, Ridolfi M, Franciosi R, Antinolfi G. The crowned dens syndrome. Evaluation with CT imaging. Radiol Med (Torino) 2007;112(2):195-207

2 Baysal T, Baysal O, Kutlu R, Karaman I, Mizrak B. The crowned dens syndrome: a rare form of calcium pyrophosphate deposition disease. Eur Radiol 2000;10(6):1003-1005

3 Salaffi F, Carotti M, Guglielmi G, Passarini G, Grassi W. The crowned dens syndrome as a cause of neck pain: clinical and computed tomography study in patients with calcium pyrophosphate dihydrate deposition disease. Clin Exp Rheumatol 2008;26(6):1040-1046

4 Fenoy AJ, Menezes AH, Donovan KA, Kralik SF. Calcium pyrophosphate dihydrate crystal deposition in the craniovertebral junction. J Neurosurg Spine 2008;8(1):22-29

5 Mavrogenis AF, Papagelopoulos PJ, Korres DS. Cervical myelopathy from calcium pyrophosphate dihydrate crystal deposition: a case report. Eur J Orthop Surg Traumatol 2010;20(6):483-487

6 Siau K, Lee M, Laversuch CJ. Acute pseudogout of the neck-the crowned dens syndrome: 2 case reports and review of the literature. Rheumatol Int 2011;31(1):85-88 Review

7 Unlu Z, Tarhan S, Ozmen EM. An idiopathic case of calcium pyrophosphate dihydrate crystal deposition disease with crowned dens syndrome in a young patient. South Med J 2009;102(9):949-951

8 Schreiter NF, Pflugmacher R, Schröder R. [CPPD-deposits-an important differential diagnosis in the retro-odontoid space in older men]. Rofo 2007;179(8):856-858

9 Ryan LM, McCarty DJ, Koopman WJ. Calcium pyrophosphate crystal deposition disease; pseudogout; articular chondrocalcinosis. In: Arthritis and Allied Conditions. 12th ed. Philadelphia, PA: Lea and Febiger; 2001:1835-1855

10 Finckh A, Van Linthoudt D, Duvoisin B, Bovay P, Gerster JC. The cervical spine in calcium pyrophosphate dihydrate deposition disease. A prevalent case-control study. J Rheumatol 2004;31(3):545-549

11 Resnick D, Pineda C. Vertebral involvement in calcium pyrophosphate dihydrate crystal deposition disease. Radiographic-pathological correlation. Radiology 1984;153(1):55-60

12 Dirheimer Y, Wackenheim C, Dietemann JL. Calcification of the transverse ligament in calcium dihydrate deposition disease (CPPD). Neuroradiology 1985;27(1):87

13 Bouvet JP, le Parc JM, Michalski B, Benlahrache C, Auquier L. Acute neck pain due to calcifications surrounding the odontoid process: the crowned dens syndrome. Arthritis Rheum 1985;28(12):1417-1420

14 Mwaka ES, Yayama T, Uchida K, et al. Calcium pyrophosphate dehydrate crystal deposition in the ligamentum flavum of the cervical spine: histopathological and immunohistochemical findings. Clin Exp Rheumatol 2009;27(3):430-438

15 Resnick D, Niwayama G, Goergen TG, et al. Clinical, radiographic and pathologic abnormalities in calcium pyrophosphate dihydrate deposition disease (CPPD): pseudogout. Radiology 1977;122(1):1-15

16 Goto S, Umehara J, Aizawa T, Kokubun S. Crowned Dens syndrome. J Bone Joint Surg Am 2007;89(12):2732-2736

17 Hasegawa H, Nakajima Y, Mabuchi E, Hashiba T, Miyao Y. Retroodontoid massive calcium pyrophosphate crystal deposition-case report. Neurol Med Chir (Tokyo) 2000;40(7):387-390

18 Fye KH, Weinstein PR, Donald F. Compressive cervical myelopathy due to calcium pyrophosphate dihydrate deposition disease: report of a case and review of the literature. Arch Intern Med 1999;159(2):189-193 
19 Feydy A, Lioté F, Carlier R, Chevrot A, Drapé JL. Cervical spine and crystal-associated diseases: imaging findings. Eur Radiol 2006; 16(2):459-468

20 Steinbach LS, Resnick D. Calcium pyrophosphate dihydrate crystal deposition disease: imaging perspectives. Curr Probl Diagn Radiol 2000;29(6):209-229

21 Kakitsubata Y, Boutin RD, Theodorou DJ, et al. Calcium pyrophosphate dihydrate crystal deposition in and around the atlantoaxial joint: association with type 2 odontoid fractures in nine patients. Radiology 2000;216(1):213-219

22 Singh H, Harrop J, Schiffmacher P, Rosen M, Evans J. Ventral surgical approaches to craniovertebral junction chordomas. Neurosurgery 2010;66(3, Suppl):96-103

23 Hsu W, Wolinsky JP, Gokaslan ZL, Sciubba DM. Transoral approaches to the cervical spine. Neurosurgery 2010;66(3, Suppl):119-125

24 Tun K, Kaptanoglu E, Cemil B, Karahan ST, Esmer AF, Elhan A. A neurosurgical view of anatomical evaluation of anterior C1-C2 for safer transoral odontoidectomy. Eur Spine J 2008;17(6):853-856

25 Zünkeler B, Schelper R, Menezes AH. Periodontoid calcium pyrophosphate dihydrate deposition disease: "pseudogout" mass lesions of the craniocervical junction. J Neurosurg 1996;85(5):803-809
26 Sethi KS, Garg A, Sharma MC, Ahmad FU, Sharma BS. Cervicomedullary compression secondary to massive calcium pyrophosphate crystal deposition in the atlantoaxial joint with intradural extension and vertebral artery encasement. Surg Neurol 2007;67(2):200-203

27 Doita M, Shimomura T, Maeno K, Nishida K, Fujioka H, Kurosaka M. Calcium pyrophosphate dihydrate deposition in the transverse ligament of the atlas: an unusual cause of cervical myelopathy. Skeletal Radiol 2007;36(7):699-702

28 Lee RS, Kayser MV, Ali SY. Calcium phosphate microcrystal deposition in the human intervertebral disc. J Anat 2006;208(1):13-19

29 Van Linthoudt D, Cao D, Pazera A, et al. Calcium pyrophosphate dihydrate deposition disease in the transverse ligament of the atlas. Praxis (Bern 1994) 2004;93(12):472-475

30 Dirheimer Y, Bensimon C, Christmann D, Wackenheim C. Syndesmo-odontoid joint and calcium pyrophosphate dihydrate deposition disease (CPPD). Neuroradiology 1983;25(5):319-321

31 Kawano N, Yoshida S, Ohwada T, Yada K, Sasaki K, Matsuno T. Cervical radiculomyelopathy caused by deposition of calcium pyrophosphate dihydrate crystals in the ligamenta flava. Case report. J Neurosurg 1980;52(2):279-283

\section{Commentary}

\author{
Jefferson R. Wilson, ${ }^{1}$ Michael G. Fehlings ${ }^{1}$ \\ ${ }^{1}$ Division of Neurosurgery and Spinal Program, University of Toronto, \\ Toronto, Ontario, Canada
}

In this article, the authors report on their management of an elderly patient with a rare form of cervical compressive myelopathy secondary to retro-odontoid calcium pyrophosphate dehydrate (CPPD) deposition. With an overall goal of achieving decompression of the spinal cord, while minimizing the risk of serious morbidity to the patient, the authors chose to perform a transoral aspiration of the associated epidural cystic mass. Although the patient's immediate postoperative course was complicated by respiratory distress syndrome, the authors reported good clinical and radiological outcomes at follow-up.

The presented case presents a variety of challenges both at a disease and patient level. Although the deposition of CPPD is a common cause of peripheral joint arthropathy, it is a rare cause of cervical myelopathy. ${ }^{1,2}$ As a result, in comparison to cervical spondylotic myelopathy or myelopathy secondary to ossification of the posterior longitudinal ligament, the natural history and ideal treatment approach to CPPD related myelopathy remains more poorly defined. With respect to patient factors, the advanced age of the discussed patient requires the surgeon to choose an operative approach that balances the desire for complete and permanent spinal cord decompression with the potential for complications and morbidity.

In accordance with these principles, the surgical approach selected effectively minimized the extent of potentially destabilizing tissue dissection and bony resection, obviating the need for posterior placed instrumentation. Furthermore, the cyst aspiration resulted in significant spinal cord decompression with concurrent improvement in symptoms. While ostensibly successful in this case, the described approach carries several potential limitations. First, advancing a trochar in the midline through diseased bone toward the spinal canal carries the risk of iatrogenic spinal cord injury. An option would have been to undertake an endoscopic or miniopen microsurgical resection. In addition, the chosen method would be limited to those lesions with a major cystic component. Second, the durability of the chosen treatment approach remains in question. As seen on the postoperative imaging, while the epidural lesion is reduced in size as compared with preoperatively, it does remain present. Over time, potential reaccumulation of cyst contents resulting in recurrent symptoms of myelopathy would necessitate the revisiting of potential surgical options. Even if a similar, minimally invasive approach is used, this would expose an even older patient to the risks of repeat surgery and hospital admission. Moreover, CPPD is often associated with degenerative atlantoaxial instability, and continued motion at $\mathrm{C} 1-\mathrm{C} 2$ could also predispose the patient to recurrent cyst formation. That said, at 6 months follow-up, the patient remained clinically and radiologically stable, indicating that the chosen treatment approach may be sufficient throughout the long term.

In summary, the authors are to be congratulated for their innovative approach to the treatment of a rare form of myelopathy in an elderly patient. Although this approach may not be suitable for a younger individual, due to potential concerns surrounding durability, it achieved the goals of attaining cord decompression while minimizing patient harm. With the overall aging of the population, we are likely to see an expanded use of similar minimally invasive techniques when treating spinal disorders amongst the elderly in the coming years.

\section{References}

1 Goto S, Umehara J, Aizawa T, Kokubun S. Crowned Dens syndrome. J Bone Joint Surg Am 2007;89(12):2732-2736

2 Lin SH, Hsieh ET, Wu TY, Chang CW. Cervical myelopathy induced by pseudogout in ligamentum flavum and retro-odontoid mass: a case report. Spinal Cord 2006;44(11):692-694 


\section{Editorial Perspective}

EBSJ welcomes the case presentation and the thoughtfully rendered concerns about the technique described by Drs. Wilson and Fehlings. The main purpose of including this article rested in the increasing number of elderly patients who present with disabilities secondary to severe spinal disorders, usually with considerable delays in recognition. The crowned dens syndrome is an example. By showing pertinent images of this case, recognition of this condition -or other storage diseases-will hopefully increase and treatment options will be discussed more knowingly. EBSJ does not endorse the technique described because no conclusions can be drawn from a single case, especially as disease recurrence is a very distinct possibility, as pointed out by Drs. Wilson and Fehlings. Furthermore, this patient quite evidently has diffuse subaxial spondylosis with likely contributions on healthy spinal cord function, rendering determination of clinical success over time quite difficult. 